\title{
Cluster Synchronization of Time-Varying Delays Coupled Complex Networks with Nonidentical Dynamical Nodes
}

\author{
Shuguo Wang, ${ }^{1,2}$ Hongxing Yao, ${ }^{2}$ and Mingping Sun ${ }^{2}$ \\ ${ }^{1}$ Department of Mathematics and Physics, Changzhou Campus, Hohai University, Jiangsu, \\ Changzhou 213022, China \\ ${ }^{2}$ Faculty of science, Jiangsu University, Zhenjiang, Jiangsu 212013, China
}

Correspondence should be addressed to Shuguo Wang,wsg97@163.com

Received 26 August 2011; Accepted 7 December 2011

Academic Editor: J. Biazar

Copyright (C) 2012 Shuguo Wang et al. This is an open access article distributed under the Creative Commons Attribution License, which permits unrestricted use, distribution, and reproduction in any medium, provided the original work is properly cited.

\begin{abstract}
This paper investigates a new cluster synchronization scheme in the nonlinear coupled complex dynamical networks with nonidentical nodes. The controllers are designed based on the community structure of the networks; some sufficient criteria are derived to ensure cluster synchronization of the network model. Particularly, the weight configuration matrix is not assumed to be symmetric, irreducible. The numerical simulations are performed to verify the effectiveness of the theoretical results.
\end{abstract}

\section{Introduction}

Complex networks model is used to describe various interconnected systems of real world, which have become a focal research topic and have drawn much attention from researchers working in different fields; one of the most important reasons is that most practical systems can be modeled by complex dynamical networks. Recently, the research on synchronization and dynamical behavior analysis of complex network systems has become a new and important direction in this field [1-13]; many control approaches have been developed to synchronize complex networks such as feedback control, adaptive control, pinning control, impulsive control, and intermittent control [14-21].

Cluster synchronization means that nodes in the same group synchronize with each other, but there is no synchronization between nodes in different groups [22-25]; Belykh et al. [26] investigated systems of diffusively coupled identical chaotic oscillators; an effective method to determine the possible states of cluster synchronization and ensure their stability 
is presented. The method, which may find applications in communication engineering and other fields of science and technology, is illustrated through concrete examples of coupled biological cell models. Wu and $\mathrm{Lu}$ [27] investigated cluster synchronization in the adaptive complex dynamical networks with nonidentical nodes by a local control method and a novel adaptive strategy for the coupling strengths of the networks. Ma et al. [28] proposed cluster synchronization scheme via dominant intracouplings and common intercluster couplings. Sorrentino and Ott [29] studied local cluster synchronization for bipartite systems, where no intracluster couplings (driving scheme) exist. Chen and Lu [30] investigated global cluster synchronization in networks of two clusters with inter- and intracluster couplings. Belykh et al. [31,26] studied this problem in 1D and 2D lattices of coupled identical dynamical systems. Lu et al. [32] studied the cluster synchronization of general networks with nonidentical clusters and derived sufficient conditions for achieving local cluster synchronization of networks. Recently, Wang et al. [33] considered the cluster synchronization of dynamical networks with community structure and nonidentical nodes and with identical local dynamics for all individual nodes in each community by using pinning control schemes. However, there is few theoretical result on the cluster synchronization of nonlinear coupled complex networks with time-varying delays coupling and time-varying delays in nonidentical dynamical nodes.

Motivated by the above discussions, this paper investigates cluster synchronization in the nonlinear coupled complex dynamical networks with nonidentical nodes. The controllers are designed based on the community structure of the networks; some sufficient criteria are derived to ensure cluster synchronization in nonlinear coupled complex dynamical networks with time-varying delays coupling and time-varying delays in dynamic nodes. Particularly the weight configuration matrix is not assumed to be symmetric, irreducible.

The paper is organized as follows: the network model is introduced followed by some definitions, lemmas, and hypotheses in Section 2. The cluster synchronization of the complex coupled networks is discussed in Section 3. Simulations are obtained in Section 4. Finally, in Section 5 the various conclusions are discussed.

\section{Model and Preliminaries}

The network with nondelayed and time-varying delays coupling and adaptive coupling strengths can be described by

$$
\begin{aligned}
\dot{x}_{i}(t)= & f_{\phi_{i}}\left(t, x_{i}(t), x_{i}\left(t-\tau_{\phi_{i}}(t)\right)\right)+c \sum_{j=1}^{N} a_{i j} H_{1}\left(x_{j}(t)\right) \\
& +c \sum_{j=1}^{N} b_{i j} H_{2}\left(x_{j}\left(t-\eta_{\phi_{i}}(t)\right)\right), \quad i=1,2, \ldots, N
\end{aligned}
$$

where $x_{i}(t)=\left(x_{i 1}(t), x_{i 2}(t), \ldots, x_{i n}(t)\right)^{T} \in R^{n}$ is the state vector of node $i ; f_{\phi_{i}}: R^{n} \rightarrow R^{n}$ describes the local dynamics of nodes in the $\phi_{i}$ th community. For any pair of nodes $i$ and $j$, if $\phi_{i} \neq \phi_{j}$, that is, nodes $i$ and $j$ belong to different communities, then $f_{\phi_{i}} \neq f_{\phi_{j}} \cdot \eta_{\phi_{i}}(t), \tau_{\phi_{i}}(t)$, is a time-varying delay. $H_{1}(\cdot)$ and $H_{2}(\cdot)$ are nonlinear functions. $c$ is coupling strength. $A=$ $\left(a_{i j}\right)_{N \times N}, B=\left(b_{i j}\right)_{N \times N}$ are the weight configuration matrices. If there is a connection from 
node $i$ to node $j(j \neq i)$, then the $a_{i j}>0, b_{i j}>0$ otherwise, $a_{i j}=a_{j i}=0, b_{i j}=b_{j i}=0$, and the diagonal elements of matrix $A, B$ are defined as

$$
a_{i i}=-\sum_{j=1, j \neq i}^{N} a_{j i}, \quad b_{i i}=-\sum_{j=1, j \neq i}^{N} b_{j i}, \quad i=1,2, \ldots, N .
$$

Particularly, the weight configuration matrix is not assumed to be symmetric, irreducible.

When the control inputs $u_{i}(t) \in R^{n}$ and $v_{i}(t) \in R^{n}(i=1,2, \ldots, N)$ are introduced, the controlled dynamical network with respect to network (2.1) can be written as

$$
\begin{aligned}
\dot{x}_{i}(t)= & f_{\phi_{i}}\left(t, x_{i}(t), x_{i}\left(t-\tau_{\phi_{i}}(t)\right)\right)+c \sum_{j=1}^{N} a_{i j} H_{1}\left(x_{j}(t)\right) \\
& +c \sum_{j=1}^{N} b_{i j} H_{2}\left(x_{j}\left(t-\eta_{\phi_{i}}(t)\right)\right)+u_{i}(t), \quad \phi_{i}(t) \in \bar{J}_{\phi_{i^{\prime}}} \\
\dot{x}_{i}(t)= & f_{\phi_{i}}\left(t, x_{i}(t), x_{i}\left(t-\tau_{\phi_{i}}(t)\right)\right)+c \sum_{j=1}^{N} a_{i j} H_{1}\left(x_{j}(t)\right) \\
& +c \sum_{j=1}^{N} b_{i j} H_{2}\left(x_{j}\left(t-\eta_{\phi_{i}}(t)\right)\right)-v_{i}(t), \quad \phi_{i}(t) \in J_{\phi_{i}}-\bar{J}_{\phi_{i}},
\end{aligned}
$$

where $J_{\phi_{i}}$ denotes all the nodes in the $\phi_{i}$ th community and $\bar{J}_{\phi_{i}}$ represents the nodes in the $\phi_{i}$ th community which have direct links with the nodes in other communities.

The study presents the mathematical definition of the cluster synchronization.

Let $\left\{C_{1}, C_{2}, \ldots, C_{m}\right\}$ denote $m(2 \leq m \leq N)$ communities of the networks and $\bigcup_{i=1}^{m} C_{i}=$ $\{1,2, \ldots N\}$. If node $i$ belongs to the $j$ th community, then we denote $\phi_{i}=j$. We employ $f_{i}(\cdot)$ to represent the local dynamics of all nodes in the $i$ th community. Let $s_{i}(t)$ be the solution of the system $\dot{s}_{i}(t)=f_{\phi_{i}}\left(t, s_{i}(t), s_{i}\left(t-\tau_{\phi_{i}}(t)\right)\right),(i=1,2, \ldots, m)$ where $\lim _{t \rightarrow \infty}\left\|s_{i}(t)-s_{j}(t)\right\| \neq 0(i \neq j)$; the set $S=\left\{s_{1}(t), s_{2}(t), \ldots, s_{m}(t)\right\}$ is used as the cluster synchronization manifold for network (2.3). Cluster synchronization can be realized if and only if the manifold $S$ is stable.

Definition 2.1 (see [19]). The error variables as $e_{i}(t)=x_{i}(t)-s_{\phi_{i}}(t)$ for $i=1,2, \ldots, N$, where $s_{\phi_{i}}(t)$ satisfies $\dot{s}_{\phi_{i}}(t)=f_{\phi_{i}}\left(t, s_{\phi_{i}}(t), s_{\phi_{i}}\left(t-\tau_{\phi_{i}}(t)\right)\right)$.

Definition 2.2 (see [19]). Let $\{1,2, \ldots, N\}$ be the $N$ nodes of the network and $\left\{C_{1}, C_{2}, \ldots, C_{m}\right\}$ be the $m$ communities, respectively. A network with $m$ communities is said to realize cluster synchronization if $\lim _{t \rightarrow \infty} e_{i}(t)=0$ and $\lim _{t \rightarrow \infty}\left\|x_{i}(t)-x_{j}(t)\right\| \neq 0$ for $\phi_{i} \neq \phi_{j}$.

Lemma 2.3. For any two vectors $x$ and $y$, a matrix $Q>0$ with compatible dimensions, one has $2 x^{T} y \leq x^{T} Q x+y^{T} Q^{-1} y$. 
Assumption 2.4. For the vector valued function $f_{\phi_{i}}\left(t, x_{i}(t), x_{i}\left(t-\tau_{\phi_{i}}\right)\right)$, assuming that there exist positive constants $\alpha_{\phi_{i}}>0, \gamma_{\phi_{i}}>0$ such that $\mathrm{f}$ satisfies the semi-Lipschitz condition

$$
\begin{aligned}
\left(x_{i}(t)-\right. & \left.y_{i}(t)\right)^{T}\left(f_{\phi_{i}}\left(t, x_{i}(t), x_{i}\left(t-\tau_{\phi_{i}}\right)\right)-f_{\phi_{i}}\left(t, y_{i}(t), y_{i}\left(t-\tau_{\phi_{i}}\right)\right)\right) \\
\leq & \alpha_{\phi_{i}}\left(x_{i}(t)-y_{i}(t)\right)^{T}\left(x_{i}(t)-y_{i}(t)\right) \\
& +\gamma_{\phi_{i}}\left(x_{i}\left(t-\tau_{\phi_{i}}\right)-y_{i}\left(t-\tau_{\phi_{i}}\right)\right)^{T}\left(x_{i}\left(t-\tau_{\phi_{i}}\right)-y_{i}\left(t-\tau_{\phi_{i}}\right)\right),
\end{aligned}
$$

for all $x, y \in R^{n}$ and $\tau_{\phi_{i}}(t) \geq 0 . i=1,2, \ldots, N$.

Assumption 2.5. $\eta_{\phi_{i}}(t)$ and $\tau_{\phi_{i}}(t)$ is a differential function with $0 \leq \dot{\eta}_{\phi_{i}}(t) \leq \varepsilon \leq 1$ and $0 \leq$ $\dot{\tau}_{\phi_{i}}(t) \leq \varepsilon \leq 1$. Clearly, this assumption is certainly ensured if the delay $\eta_{\phi_{i}}(t)$ and $\tau_{\phi_{i}}(t)$ is constant.

Assumption 2.6. [34] (Global Lipschitz Condition)]

Suppose that there exist nonnegative constants $\vartheta, \beta$, for all $t \in R_{+}$, such that for any time-varying vectors $x(t), y(t) \in R^{n}$

$$
\left\|H_{1}(x)-H_{1}(y)\right\| \leq \vartheta\|x-y\|, \quad\left\|H_{2}(x)-H_{2}(y)\right\| \leq \beta\|x-y\|,
$$

where || || denotes the 2-norm throughout the paper.

\section{Main Results}

In this section, a control scheme is developed to synchronize a delayed complex network with nonidentical nodes to any smooth dynamics $s_{\phi_{i}}(t)$. Let synchronization errors $e_{i}(t)=$ $x_{i}(t)-s_{\phi_{i}}(t)$ for $i=1,2, \ldots, N$, according to system (2.1), the error dynamical system can be derived as

$$
\begin{aligned}
\dot{e}_{i}(t)= & \tilde{f}_{\phi_{i}}\left(t, x_{i}(t), x_{i}\left(t-\tau_{\phi_{i}}(t)\right)\right)+c \sum_{j=1}^{N} a_{i j}\left[H_{1}\left(x_{j}(t)\right)-H_{1}\left(s_{\phi_{i}}(t)\right)\right] \\
& +c \sum_{j=1}^{N} b_{i j}\left[H_{2}\left(x_{j}\left(t-\eta_{\phi_{i}}(t)\right)\right)-H_{2}\left(s_{\phi_{i}}\left(t-\eta_{\phi_{i}}(t)\right)\right)\right]+\sum_{i=1}^{N} a_{i j} H_{1}\left(s_{\phi_{i}}(t)\right) \\
& +\sum_{i=1}^{N} b_{i j} H_{2}\left(s_{\phi_{i}}\left(t-\eta_{\phi_{i}}(t)\right)\right)+u_{i}(t), \quad \phi_{i}(t) \in \bar{J}_{\phi_{i^{\prime}}} \\
\dot{e}_{i}(t)= & \tilde{f}_{\phi_{i}}\left(t, x_{i}(t), x_{i}\left(t-\tau_{\phi_{i}}(t)\right)\right)+c \sum_{j=1}^{N} a_{i j}\left[H_{1}\left(x_{j}(t)\right)-H_{1}\left(s_{\phi_{i}}(t)\right)\right] \\
& +c \sum_{j=1}^{N} b_{i j}\left[H_{2}\left(x_{j}\left(t-\eta_{\phi_{i}}(t)\right)\right)-H_{2}\left(s_{\phi_{i}}\left(t-\eta_{\phi_{i}}(t)\right)\right)\right] \\
& -v_{i}(t), \quad \phi_{i}(t) \in J_{\phi_{i}}-\bar{J}_{\phi_{i^{\prime}}}
\end{aligned}
$$

where $\tilde{f}_{\phi_{i}}\left(t, x_{i}(t), x_{i}\left(t-\tau_{\phi_{i}}(t)\right)\right)=f_{\phi_{i}}\left(t, x_{i}(t), x_{i}\left(t-\tau_{\phi_{i}}(t)\right)\right)-f_{\phi_{i}}\left(t, s_{\phi_{i}}(t), s_{\phi_{i}}\left(t-\tau_{\phi_{i}}(t)\right)\right)$ for $i=$ $1,2, \ldots, N$. 
Journal of Applied Mathematics

According to the diffusive coupling condition (2.2) of the matrix $A, B$ we have

$$
c \sum_{i=1}^{N} a_{i j} H_{1}\left(s_{\phi_{i}}(t)\right)+c \sum_{i=1}^{N} b_{i j} H_{2}\left(s_{\phi_{i}}\left(t-\eta_{\phi_{i}}(t)\right)\right)=0, \quad i \in J_{\phi_{i}}-\bar{J}_{\phi_{i}} .
$$

On the basis of this property, for achieving cluster synchronization, we design controllers as follows:

$$
u_{i}(t)= \begin{cases}-c \sum_{i=1}^{N} a_{i j} H_{1}\left(s_{\phi_{i}}(t)\right)-c \sum_{i=1}^{N} b_{i j} H_{2}\left(s_{\phi_{i}}\left(t-\eta_{\phi_{i}}(t)\right)\right)-d_{i} e_{i}(t), & i \in \bar{J}_{\phi_{i^{\prime}}} \\ v_{i}(t)=d_{i} e_{i}(t), & i \in J_{\phi_{i}}-\bar{J}_{\phi_{i^{\prime}}},\end{cases}
$$

where $\dot{d}_{i}=k_{i} e_{i}^{T}(t) e_{i}(t)$.

Theorem 3.1. Suppose assumptions 2.4-2.5 hold. Consider the network (2.1) via control law (3.3). If the following conditions hold:

$$
\alpha+\vartheta c \lambda_{\max }(Q)+\frac{1}{2} \beta^{2} c^{2} \lambda_{\max }\left(P P^{T}\right)+\frac{1}{1-\varepsilon}\left(\gamma+\frac{1}{2}\right)<d,
$$

where $\alpha=\max \left(\alpha_{\phi_{1}}, \alpha_{\phi_{2}}, \ldots, \alpha_{\phi_{m}}\right), \gamma=\max \left(\gamma_{\phi_{1}}, \gamma_{\phi_{2}}, \ldots, \gamma_{\phi_{m}}\right)$. Then, the systems (2.3) is cluster synchronization.

Proof. Construct the following Lyapunov functional:

$$
\begin{aligned}
V(t)= & \frac{1}{2} \sum_{i=1}^{N} e_{i}^{T}(t) e_{i}(t)+\frac{\gamma}{1-\varepsilon} \int_{t-\tau_{\phi_{i}}(t)}^{t} \sum_{i=1}^{N} e_{i}^{T}(\theta) e_{i}(\theta) d \theta \\
& +\frac{1}{2(1-\varepsilon)} \int_{t-\eta_{\phi_{i}}(t)}^{t} \sum_{i=1}^{N} e_{i}^{T}(\theta) e_{i}(\theta) d \theta+\frac{1}{2} \sum_{i=1}^{N} \frac{\left(d_{i}-d\right)^{2}}{k_{i}} .
\end{aligned}
$$

Calculating the derivative of $V(t)$, we have

$$
\begin{aligned}
\dot{V}(t)= & \sum_{i=1}^{N} e_{i}^{T}(t) \dot{e}_{i}(t)+\frac{1}{1-\varepsilon}\left(\gamma+\frac{1}{2}\right) \sum_{i=1}^{N} e_{i}^{T}(t) e_{i}(t)-\frac{\gamma\left(1-\dot{\tau}_{\phi_{i}}(t)\right)}{1-\varepsilon} \sum_{i=1}^{N} e_{i}^{T}\left(t-\tau_{\phi_{i}}(t)\right) e_{i}\left(t-\tau_{\phi_{i}}(t)\right) \\
& -\frac{1-\dot{\eta}_{\phi_{i}}(t)}{2(1-\varepsilon)} \sum_{i=1}^{N} e_{i}^{T}\left(t-\eta_{\phi_{i}}(t)\right) e_{i}\left(t-\eta_{\phi_{i}}(t)\right)+\sum_{i=1}^{N}\left(d_{i}-d\right) e_{i}^{T}(t) e_{i}(t)
\end{aligned}
$$




$$
\begin{gathered}
=\sum_{i=1}^{N} e_{i}^{T}(t)\left\{\tilde{f}_{\phi_{i}}\left(t, x_{i}(t), x_{i}\left(t-\tau_{\phi_{i}}(t)\right)\right)+c \sum_{j=1}^{N} a_{i j}\left[H_{1}\left(x_{j}(t)\right)-H_{1}\left(s_{\phi_{i}}(t)\right)\right]\right. \\
\left.+c \sum_{j=1}^{N} b_{i j}\left[H_{2}\left(x_{j}\left(t-\eta_{\phi_{i}}(t)\right)\right)-H_{2}\left(s_{\phi_{i}}\left(t-\eta_{\phi_{i}}(t)\right)\right)\right]-d_{i} e_{i}(t)\right\} \\
+\frac{1}{1-\varepsilon}\left(\gamma+\frac{1}{2}\right) \sum_{i=1}^{N} e_{i}^{T}(t) e_{i}(t)-\frac{\gamma\left(1-\dot{\tau}_{\phi_{i}}(t)\right)}{1-\varepsilon} \sum_{i=1}^{N} e_{i}^{T}\left(t-\tau_{\phi_{i}}(t)\right) e_{i}\left(t-\tau_{\phi_{i}}(t)\right) \\
-\frac{1-\dot{\eta}_{\phi_{i}}(t)}{2(1-\varepsilon)} \sum_{i=1}^{N} e_{i}^{T}\left(t-\eta_{\phi_{i}}(t)\right) e_{i}\left(t-\eta_{\phi_{i}}(t)\right)+\sum_{i=1}^{N}\left(d_{i}-d\right) e_{i}^{T}(t) e_{i}(t) .
\end{gathered}
$$

By assumptions 2.4-2.6, we obtain

$$
\begin{aligned}
\leq & \alpha \sum_{i=1}^{N} e_{i}^{T}(t) e_{i}(t)+\gamma \sum_{i=1}^{N} e_{i}^{T}\left(t-\tau_{\phi_{i}}(t)\right) e_{i}\left(t-\tau_{\phi_{i}}(t)\right)+\vartheta_{c} \sum_{i=1}^{N} e_{i}^{T}(t) \sum_{j=1}^{N} a_{i j} e_{j}(t) \\
& +\beta c \sum_{i=1}^{N} e_{i}^{T}(t) \sum_{j=1}^{N} b_{i j} e_{j}\left(t-\eta_{\phi_{i}}(t)\right)-d_{i} \sum_{i=1}^{N} e_{i}^{T}(t) e_{i}(t) \\
& +\frac{1}{1-\varepsilon}\left(\gamma+\frac{1}{2}\right) \sum_{i=1}^{N} e_{i}^{T}(t) e_{i}(t)-\gamma \sum_{i=1}^{N} e_{i}^{T}\left(t-\tau_{\phi_{i}}(t)\right) e_{i}\left(t-\tau_{\phi_{i}}(t)\right) \\
& -\frac{1}{2} \sum_{i=1}^{N} e_{i}^{T}\left(t-\eta_{\phi_{i}}(t)\right) e_{i}\left(t-\eta_{\phi_{i}}(t)\right)+\sum_{i=1}^{N}\left(d_{i}-d\right) e_{i}^{T}(t) e_{i}(t) \\
\leq & \alpha \sum_{i=1}^{N} e_{i}^{T}(t) e_{i}(t)+\vartheta c e^{T}(A \otimes I) e+\beta c e^{T}(B \otimes I) e\left(t-\eta_{\phi_{i}}(t)\right)+\frac{1}{1-\varepsilon}\left(\gamma+\frac{1}{2}\right) \sum_{i=1}^{N} e_{i}^{T}(t) e_{i}(t) \\
& -\frac{1}{2} \sum_{i=1}^{N} e_{i}^{T}\left(t-\eta_{\phi_{i}}(t)\right) e_{i}\left(t-\eta_{\phi_{i}}(t)\right)-d e^{T}(t) e(t) .
\end{aligned}
$$

Let $e(t)=\left(e_{1}^{T}(t), e_{2}^{T}(t), \ldots, e_{N}^{T}(t)\right)^{T} \in R^{n N}, Q=(A \otimes I), P=(B \otimes I)$, where $\otimes$ represents the Kronecker product. Then

$$
\begin{aligned}
\dot{V}(t) \leq & \alpha e^{T}(t) e(t)+\vartheta c e^{T}(t) Q e(t)+\beta c e^{T}(t) P e\left(t-\eta_{\phi_{i}}(t)\right)+\frac{1}{1-\varepsilon}\left(\gamma+\frac{1}{2}\right) e^{T}(t) e(t) \\
& -\frac{1}{2} e^{T}\left(t-\eta_{\phi_{i}}(t)\right) e\left(t-\eta_{\phi_{i}}(t)\right)-d e^{T}(t) e(t) .
\end{aligned}
$$

By the Lemma 2.3, we have

$$
\begin{aligned}
& \leq \alpha e^{T}(t) e(t)+\vartheta c e^{T}(t) Q e(t)+\frac{1}{2}(\beta c)^{2} e^{T}(t) P P^{T} e(t)+\frac{1}{1-\varepsilon}\left(\gamma+\frac{1}{2}\right) e^{T}(t) e(t)-d e^{T}(t) e(t) \\
& \leq\left(\alpha+\vartheta c \lambda_{\max }(Q)+\frac{1}{2} \beta^{2} c^{2} \lambda_{\max }\left(P P^{T}\right)+\frac{1}{1-\varepsilon}\left(\gamma+\frac{1}{2}\right)-d\right) e^{T}(t) e(t)
\end{aligned}
$$


Therefore, if we have $\alpha+\vartheta_{c} \lambda_{\max }(Q)+(1 / 2) \beta^{2} c^{2} \lambda_{\max }\left(P P^{T}\right)+(1 /(1-\varepsilon))(\gamma+(1 / 2))<d$ then

$$
\dot{V}(t) \leq 0
$$

Theorem 3.1 is proved completely.

We can conclude that, for any initial values, the solutions $x_{1}(t), x_{2}(t), \ldots, x_{N}(t)$ of the system (2.3) satisfy $\lim _{t \rightarrow \infty} \sum_{k=1}^{m} \sum_{i \in C_{k}}\left\|x_{i}(t)-s_{k}(t)\right\|=0$, that is, we get the global stability of the cluster synchronization manifold $S$. Therefore, cluster synchronization in the network (2.3) is achieved under the local controllers (3.3). This completes the proof.

Corollary 3.2. When $A=0$, network (2.1) is translated into

$$
\dot{x}_{i}(t)=f_{\phi_{i}}\left(t, x_{i}(t), x_{i}\left(t-\tau_{\phi_{i}}(t)\right)\right)+c \sum_{j=1}^{N} b_{i j} H_{2}\left(x_{j}\left(t-\eta_{\phi_{i}}(t)\right)\right), \quad i=1,2, \ldots, N
$$

We design the controllers, as follows, then the complex networks can also achieve synchronization, where

$$
u_{i}(t)= \begin{cases}-c \sum_{i=1}^{N} b_{i j} H_{2}\left(s_{\phi_{i}}\left(t-\eta_{\phi_{i}}(t)\right)\right)-d_{i} e_{i}(t), & i \in \bar{J}_{\phi_{i^{\prime}}} \\ v_{i}(t)=d_{i} e_{i}(t), & i \in J_{\phi_{i}}-\bar{J}_{\phi_{i}} .\end{cases}
$$

Corollary 3.3. When $B=0$, network (2.1) is translated into

$$
\dot{x}_{i}(t)=f_{\phi_{i}}\left(t, x_{i}(t), x_{i}\left(t-\tau_{\phi_{i}}(t)\right)\right)+c \sum_{j=1}^{N} a_{i j} H_{1}\left(x_{j}(t)\right), \quad i=1,2, \ldots, N
$$

We design the controllers, as follows, then the complex networks can also achieve synchronization, where

$$
u_{i}(t)= \begin{cases}-c \sum_{i=1}^{N} a_{i j} H_{1}\left(s_{\phi_{i}}(t)\right)-d_{i} e_{i}(t), & i \in \bar{J}_{\phi_{i}} \\ v_{i}(t)=d_{i} e_{i}(t), & i \in J_{\phi_{i}}-\bar{J}_{\phi_{i}},\end{cases}
$$




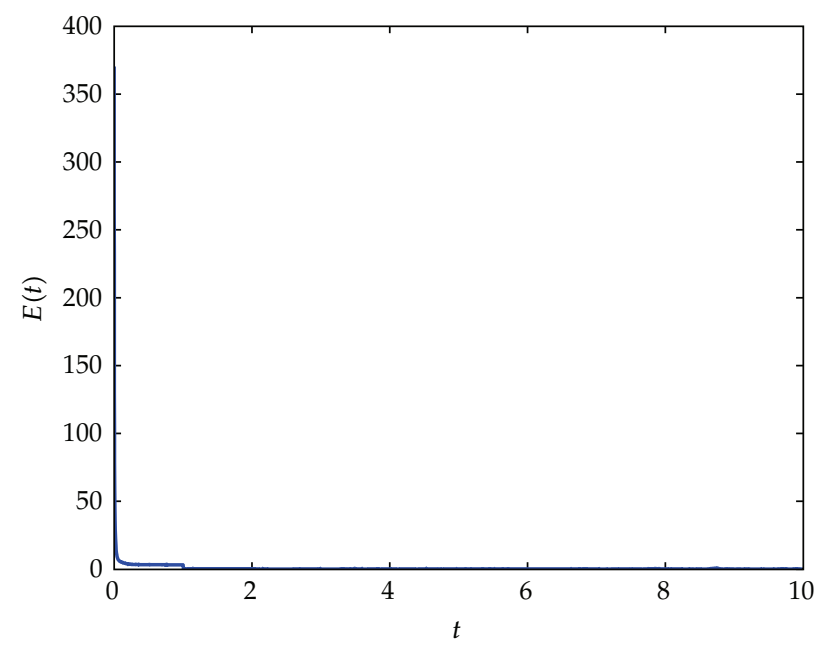

Figure 1: Time evolution of the synchronization errors $E(t)$.

\section{Illustrative Examples}

In this section, a numerical example will be given to demonstrate the validity of the synchronization criteria obtained in the previous sections. Considering the following network:

$$
\begin{aligned}
\dot{x}_{i}(t)= & f_{\phi_{i}}\left(t, x_{i}(t), x_{i}\left(t-\tau_{\phi_{i}}(t)\right)\right)+c \sum_{j=1}^{N} a_{i j} H_{1}\left(x_{j}(t)\right) \\
& +c \sum_{j=1}^{N} b_{i j} H_{2}\left(x_{j}\left(t-\eta_{\phi_{i}}(t)\right)\right)+u_{i}(t), \quad \phi_{i}(t) \in \bar{J}_{\phi_{i^{\prime}}} \\
\dot{x}_{i}(t)= & f_{\phi_{i}}\left(t, x_{i}(t), x_{i}\left(t-\tau_{\phi_{i}}(t)\right)\right)+c \sum_{j=1}^{N} a_{i j} H_{1}\left(x_{j}(t)\right) \\
& +c \sum_{j=1}^{N} b_{i j} H_{2}\left(x_{j}\left(t-\eta_{\phi_{i}}(t)\right)\right)-v_{i}(t), \quad \phi_{i}(t) \in J_{\phi_{i}}-\bar{J}_{\phi_{i}}, i=1,2, \ldots, N,
\end{aligned}
$$

where $x_{i}(t)=\left(x_{i 1}(t), x_{i 2}(t), x_{i 3}(t)\right)^{T}, f_{1}\left(t, x_{i}(t), x_{i}\left(t-\tau_{1}(t)\right)\right)=D_{1} x_{i}(t)+h_{11}\left(x_{i}(t)\right)+h_{12}\left(x_{i}(t-\right.$ $\left.\left.\tau_{1}(t)\right)\right), f_{2}\left(t, x_{i}(t), x_{i}\left(t-\tau_{2}(t)\right)\right)=D_{2} x_{i}(t)+h_{21}\left(x_{i}(t)\right)+h_{22}\left(x_{i}\left(t-\tau_{2}(t)\right)\right)+V, f_{3}\left(t, x_{i}(t), x_{i}(t-\right.$ $\left.\left.\tau_{3}(t)\right)\right)=D_{3} x_{i}(t)+h_{31}\left(x_{i}(t)\right)+h_{32}\left(x_{i}\left(t-\tau_{3}(t)\right)\right) . k_{1}=k_{2}=\cdots=k_{N}=10, c=1, H_{1}(x)=$ $\sin x, H_{2}(x)=\cos x$.

In simulation, we choose $h_{11}\left(x_{i}\right)=\left(0,-x_{i 1} x_{i 3}, x_{i 1} x_{i 2}\right)^{T}, h_{12}\left(x_{i}\right)=\left(0,5 x_{i 2}, 0\right)^{T}, h_{21}\left(x_{i}\right)=$ $\left(0,0, x_{i 1} x_{i 3}\right)^{T}, h_{22}\left(x_{i}\right)=\left(x_{i 1}, 0,0\right)^{T}, V=[0,0,0.2]^{T}, h_{31}\left(x_{i}\right)=\left(3.247\left(\left|x_{i 1}+1\right|-\mid x_{i 1}-\right.\right.$ 


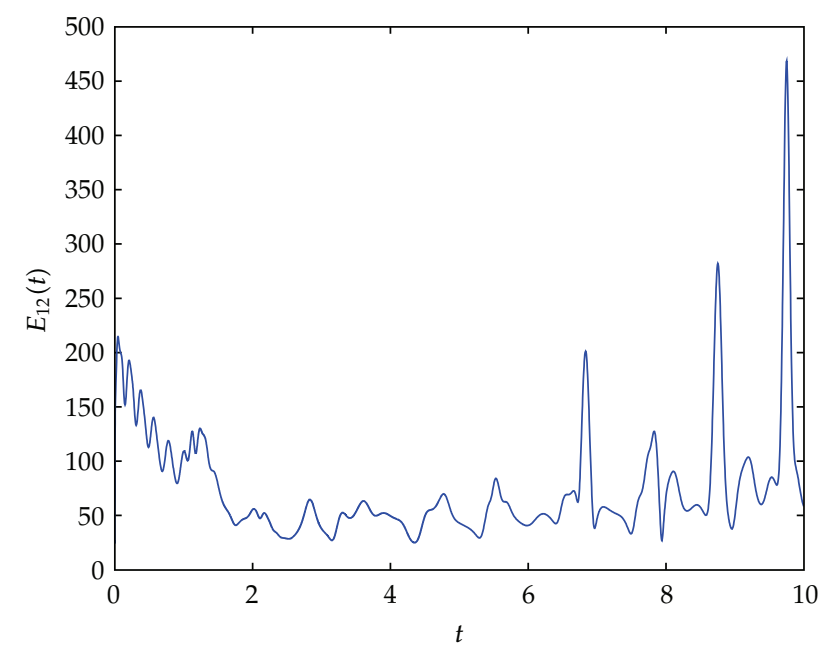

Figure 2: Time evolution of the synchronization errors $E_{12}(t)$.

$1 \mid), 0,0)^{T}, h_{32}\left(x_{i}\right)=\left(0,0,-3.906 \sin \left(0.5 x_{i 1}\right)\right)^{T}, \tau_{1}(t)=e^{t} /\left(1+e^{t}\right), \tau_{2}(t)=2 e^{t} /\left(1+e^{t}\right), \tau_{3}(t)=$ $0.5 e^{t} /\left(1+e^{t}\right)$,

$$
D_{1}=\left[\begin{array}{ccc}
-10 & 10 & 0 \\
28 & 4 & 0 \\
0 & 0 & -\frac{8}{3}
\end{array}\right], \quad D_{2}=\left[\begin{array}{ccc}
0 & -1 & -1 \\
1 & 0.2 & 0 \\
0 & 0 & -1.2
\end{array}\right], \quad D_{3}=\left[\begin{array}{ccc}
-2.169 & 10 & 0 \\
1 & -1 & 1 \\
0 & -19.53 & -0.1636
\end{array}\right]
$$

Taking the weight configuration coupling matrices

$$
A=B=\left[\begin{array}{cccccc}
-2 & 1 & 0 & 0 & 0 & 1 \\
-1 & -2 & 1 & 0 & 0 & 0 \\
0 & 1 & -2 & 1 & 0 & 0 \\
0 & 0 & 1 & -2 & 1 & 0 \\
0 & 0 & 0 & 1 & -2 & 1 \\
1 & 0 & 0 & 0 & 1 & -2
\end{array}\right]
$$

The following quantities are utilized to measure the process of cluster synchronization

$$
\begin{gathered}
E(t)=\sum_{i=1}^{N}\left\|x_{i}(t)-s_{\phi_{i}}(t)\right\|, \\
E_{12}(t)=\left\|x_{u}(t)-x_{v}(t)\right\|, \quad u \in C_{1}, v \in C_{2}, \\
E_{13}(t)=\left\|x_{u}(t)-x_{v}(t)\right\|, \quad u \in C_{1}, v \in C_{3}, \\
E_{23}(t)=\left\|x_{u}(t)-x_{v}(t)\right\|, \quad u \in C_{2}, v \in C_{3},
\end{gathered}
$$

where $E(t)$ is the error of cluster synchronization for this controlled network (2.2); $E_{12}(t)$, $E_{13}(t)$, and $E_{23}(t)$ are the errors between two communities; cluster synchronization is 


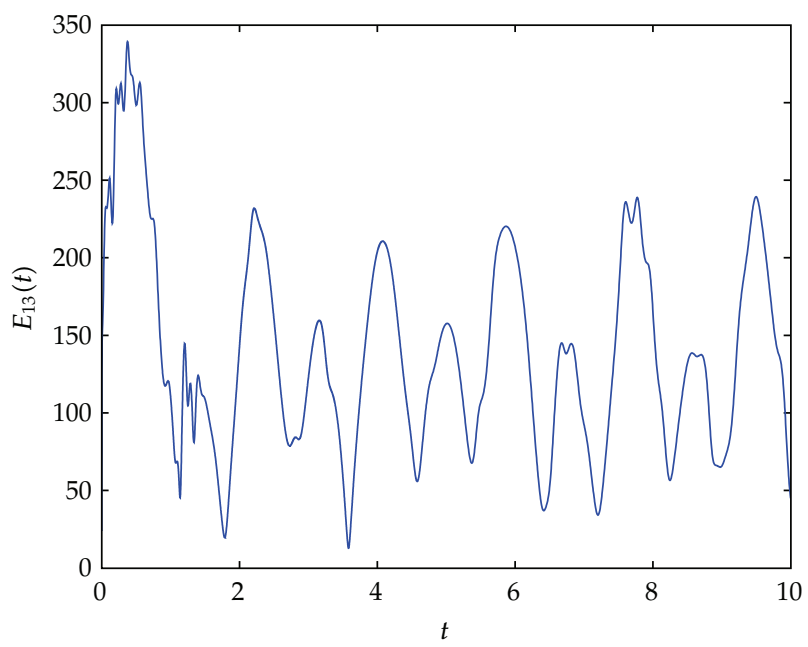

Figure 3: Time evolution of the synchronization errors $E_{13}(t)$.

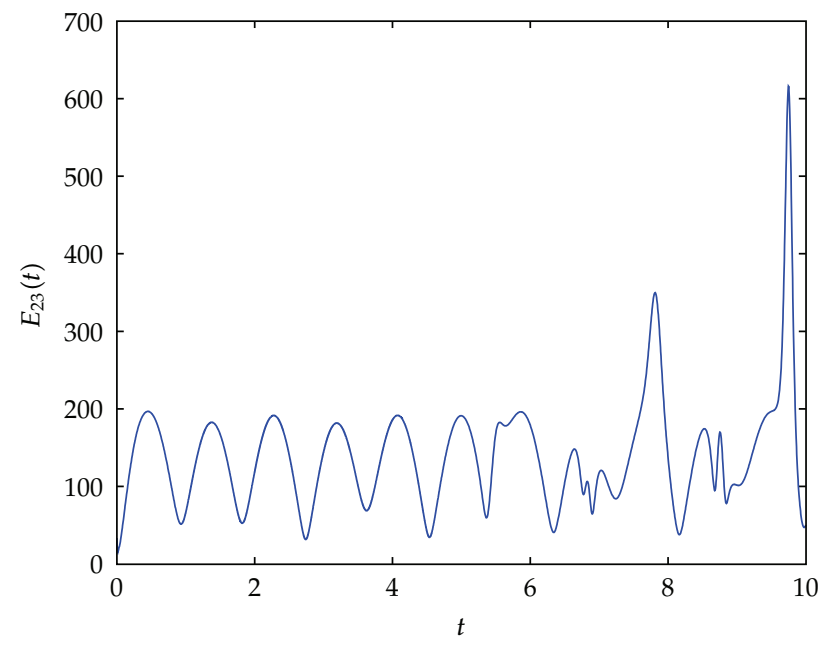

Figure 4: Time evolution of the synchronization errors $E_{23}(t)$.

achieved if the synchronization error $E(t)$ converges to zero and $E_{12}(t), E_{13}(t)$ and $E_{23}(t)$ do not as $t \rightarrow \infty$. Simulation results are given in Figures 1, 2, 3, and 4. From the Figures $1-4$, we see the time evolution of the synchronization errors. The numerical results show that Theorem 3.1 is effective.

\section{Conclusions}

The problems of cluster synchronization and adaptive feedback controller for the nonlinear coupled complex networks are investigated. The weight configuration matrix is not assumed to be symmetric, irreducible. It is shown that cluster synchronization can be realized via adaptive feedback controller. The study showed that the use of simple control law helps 
to derive sufficient criteria which ensure that nodes in the same group synchronize with each other, but there is no synchronization between nodes in different groups is derived. Particularly the synchronization criteria are independent of time delay. The developed techniques are applied three complex community networks which are synchronized to different chaotic trajectories. Finally, the numerical simulations were performed to verify the effectiveness of the theoretical results.

\section{Acknowledgments}

This research is partially supported by the National Nature Science Foundation of China (no. 70871056) and by the Six Talents Peak Foundation of Jiangsu Province.

\section{References}

[1] J. Cao, P. Li, and W. Wang, "Global synchronization in arrays of delayed neural networks with constant and delayed coupling," Physics Letters A, vol. 353, no. 4, pp. 318-325, 2006.

[2] M. Chen, "Some simple synchronization criteria for complex dynamical networks," IEEE Transactions on Circuits and Systems II: Express Briefs, vol. 53, no. 11, pp. 1185-1189, 2006.

[3] H. Gao, J. Lam, and G. Chen, "New criteria for synchronization stability of general complex dynamical networks with coupling delays," Physics Letters A, vol. 360, no. 2, pp. 263-273, 2006.

[4] Y. He, Q.-G. Wang, and W.-X. Zheng, "Global robust stability for delayed neural networks with polytopic type uncertainties," Chaos, Solitons and Fractals, vol. 26, no. 5, pp. 1349-1354, 2005.

[5] J. Zhou, L. Xiang, and Z. Liu, "Global synchronization in general complex delayed dynamical networks and its applications," Physica A, vol. 385, no. 2, pp. 729-742, 2007.

[6] X. P. Han, J.-A. Lu, and G. R. Chen, "Nonlinear integral synchronization of ring networks," Computers \& Mathematics with Applications, vol. 55, no. 4, pp. 808-818, 2008.

[7] S. Y. Xu and Y. Yang, "Synchronization for a class of complex dynamical networks with time-delay," Communications in Nonlinear Science and Numerical Simulation, vol. 14, no. 8, pp. 3230-3238, 2009.

[8] Z. Jia, H. Wang, J. Wang, and Y. Li, “The weighted identification of a bipartite-graph complex dynamical network based on adaptive synchronization," Control Theory and Applications, vol. 27, no. 1, pp. 107-110, 2010.

[9] L. Wang, H.-P. Dai, H. Dong, Y.-H. Shen, and Y.-X. Sun, "Adaptive synchronization of weighted complex dynamical networks with coupling time-varying delays," Physics Letters A, vol. 372, no. 20, pp. 3632-3639, 2008.

[10] D. H. Ji, J. H. Park, W. J. Yoo, S. C. Won, and S. M. Lee, "Synchronization criterion for Lur'e type complex dynamical networks with time-varying delay," Physics Letters A, vol. 374, no. 10, pp. 12181227, 2010.

[11] K. Yuan, "Robust synchronization in arrays of coupled networks with delay and mixed coupling," Neurocomputing, vol. 72, no. 4-6, pp. 1026-1031, 2009.

[12] T. Liu, J. Zhao, and D. J. Hill, "Synchronization of complex delayed dynamical networks with nonlinearly coupled nodes," Chaos, Solitons and Fractals, vol. 40, no. 3, pp. 1506-1519, 2009.

[13] W. L. Guo, F. Austin, and S. H. Chen, "Global synchronization of nonlinearly coupled complex networks with non-delayed and delayed coupling," Communications in Nonlinear Science and Numerical Simulation, vol. 15, no. 6, pp. 1631-1639, 2010.

[14] P. DeLellis, M. diBernardo, and F. Garofalo, "Novel decentralized adaptive strategies for the synchronization of complex networks," Automatica, vol. 45, no. 5, pp. 1312-1318, 2009.

[15] S. Zheng, S. Wang, G. Dong, and Q. Bi, "Adaptive synchronization of two nonlinearly coupled complex dynamical networks with delayed coupling," Communications in Nonlinear Science and Numerical Simulation, vol. 17, no. 1, pp. 284-291, 2012.

[16] M. Porfiri and M. di Bernardo, "Criteria for global pinning-controllability of complex networks," Automatica, vol. 44, no. 12, pp. 3100-3106, 2008. 
[17] F. Sorrentino, M. D. Bernardo, F. Garofalo, and G. R. Chen, “Controllability of complex networks via pinning," Physical Review E, vol. 75, no. 4, Article ID 046103, 6 pages, 2007.

[18] W. Xia and J. Cao, "Pinning synchronization of delayed dynamical networks via periodically intermittent control," Chaos, vol. 19, no. 1, Article ID 013120, 8 pages, 2009.

[19] S. Cai, Z. Liu, F. Xu, and J. Shen, "Periodically intermittent controlling complex dynamical networks with time-varying delays to a desired orbit," Physics Letters A, vol. 373, no. 42, pp. 3846-3854, 2009.

[20] A. Khadra, X. Z. Liu, and X. Shen, "Impulsively synchronizing chaotic systems with delay and applications to secure communication," Automatica, vol. 41, no. 9, pp. 1491-1502, 2005.

[21] W. Sun, T. Hu, Z. Chen, S. Chen, and L. Xiao, "Impulsive synchronization of a general nonlinear coupled complex network," Communications in Nonlinear Science and Numerical Simulation, vol. 16, no. 11, pp. 4501-4507, 2011.

[22] M. I. Rabinovich, J. J. Torres, P. Varona, R. Huerta, and P. Weidman, “Origin of coherent structures in a discrete chaotic medium," Physical Review E, vol. 60, no. 2, pp. R1130-R1133, 1999.

[23] D. H. Zanette and A. S. Mikhailov, "Mutual synchronization in ensembles of globally coupled neural networks," Physical Review E, vol. 58, no. 1, pp. 872-875, 1998.

[24] I. Belykh, V. Belykh, K. Nevidin, and M. Hasler, "Persistent clusters in lattices of coupled nonidentical chaotic systems," Chaos, vol. 13, no. 1, pp. 165-178, 2003.

[25] V. N. Belykh, I. V. Belykh, M. Hasler, and K. V. Nevidin, "Cluster synchronization in three-dimensional lattices of diffusively coupled oscillators," International Journal of Bifurcation and Chaos, vol. 13, no. 4, pp. 755-779, 2003.

[26] N. V. Belykh, I. V. Belykh, and E. Mosekilde, "Cluster synchronization modes in an ensemble of coupled chaotic oscillators," Physical Review E, vol. 63, no. 3, part 2, Article ID 036216, 2001.

[27] X. Wu and H. Lu, "Cluster synchronization in the adaptive complex dynamical networks via a novel approach," Physics Letters A, vol. 375, no. 14, pp. 1559-1565, 2011.

[28] Z. Ma, Z. Liu, and G. Zhang, "A new method to realize cluster synchronization in connected chaotic networks," Chaos, vol. 16, no. 2, Article ID 023103, 9 pages, 2006.

[29] F. Sorrentino and E. Ott, "Network synchronization of groups," Physical Review E, vol. 76, no. 5, Article ID 056114, 10 pages, 2007.

[30] L. Chen and J. Lu, "Cluster synchronization in a complex dynamical network with two nonidentical clusters," Journal of Systems Science E Complexity, vol. 21, no. 1, pp. 20-33, 2008.

[31] V. N. Belykh, I. V. Belykh, and M. Hasler, "Hierarchy and stability of partially synchronous oscillations of diffusively coupled dynamical systems," Physical Review E, vol. 62, no. 5, pp. 6332-6345, 2000.

[32] W. L. Lu, B. Liu, and T. Chen, "Cluster synchronization in networks of distinct groups of maps," European Physical Journal B, vol. 77, no. 2, pp. 257-264, 2010.

[33] K. Wang, X. Fu, and K. Li, "Cluster synchronization in community networks with nonidentical nodes," Chaos. An Interdisciplinary Journal of Nonlinear Science, vol. 19, no. 2, Article ID 023106, 10 pages, 2009.

[34] H. Liu, J. Chen, J. A. Lu, and M. Cao, “Generalized synchronization in complex dynamical networks via adaptive couplings," Physica A, vol. 389, no. 8, pp. 1759-1770, 2010. 


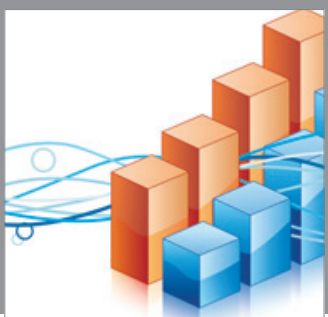

Advances in

Operations Research

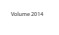

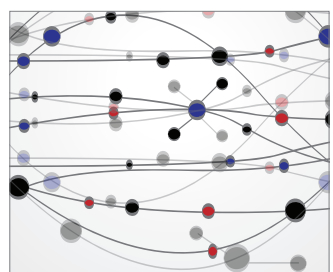

\section{The Scientific} World Journal
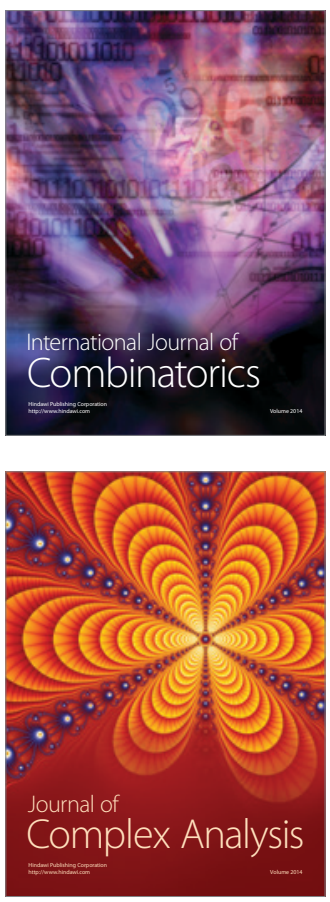

International Journal of

Mathematics and

Mathematical

Sciences
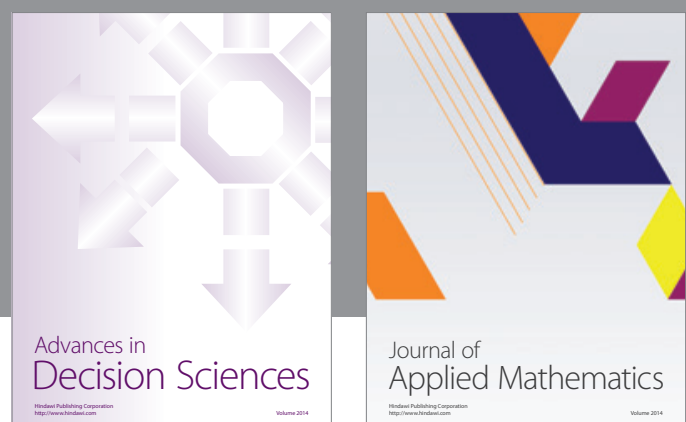

Journal of

Applied Mathematics
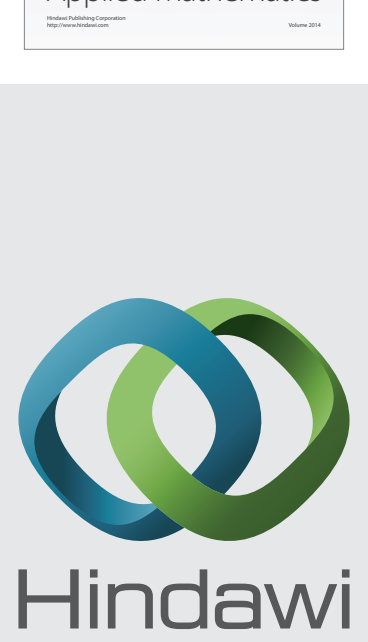

Submit your manuscripts at http://www.hindawi.com
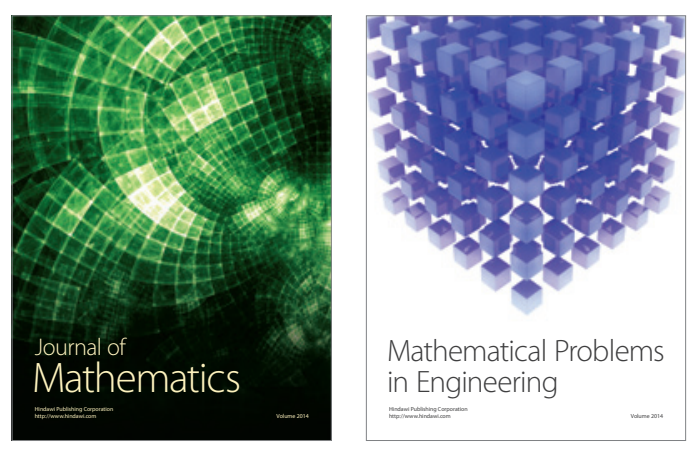

Mathematical Problems in Engineering
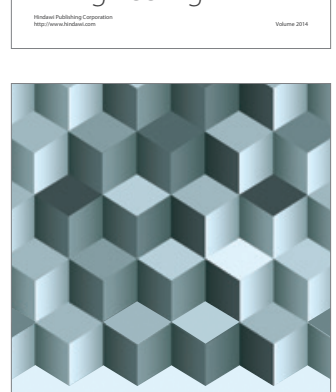

Journal of

Function Spaces
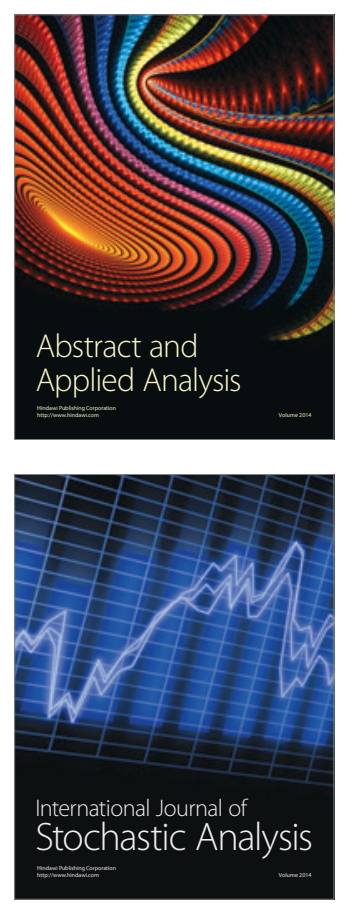

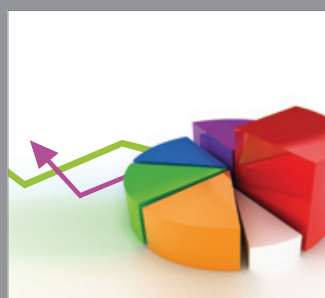

ournal of

Probability and Statistics

Promensencen
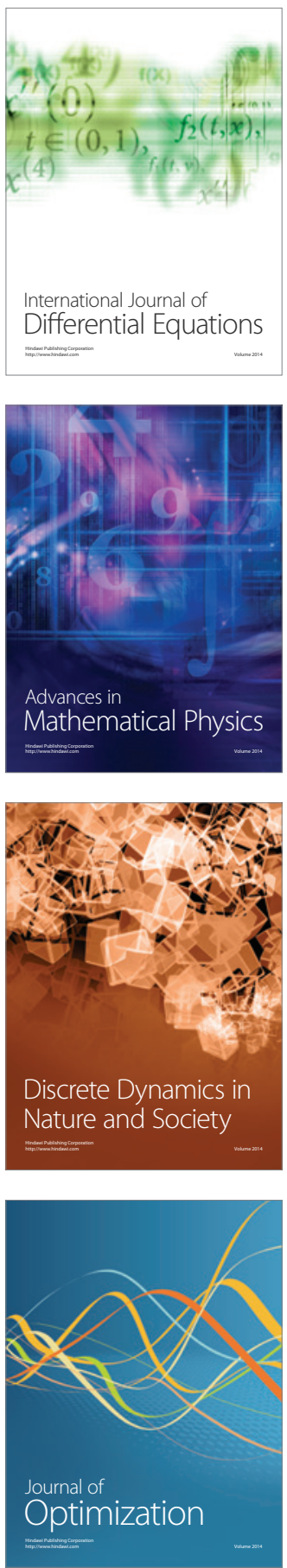